\title{
Correlation of Suicidal Thoughts and Suicide Attempts in Patients with Multiple Sclerosis
}

\author{
Payam Sariaslani ${ }^{1,2}$, Parvin Ghanbari ${ }^{3}$ and Saeid Komasi (iD ${ }^{4, *}$ \\ ${ }^{1}$ Student Research Committee, Kermanshah University of Medical Sciences, Kermanshah, Iran \\ ${ }^{2}$ Department of Neurology, Imam Reza Hospital, Kermanshah University of Medical Sciences, Kermanshah, Iran \\ ${ }^{3}$ Cardiac Rehabilitation Center, Imam Ali Hospital, Kermanshah University of Medical Sciences, Kermanshah, Iran \\ ${ }^{4}$ Department of Neuroscience and Psychopathology Research, Mind GPS Institute, Kermanshah, Iran \\ "Corresponding author: Department of Neuroscience and Psychopathology Research, Mind GPS Institute, Nasr Boulevard, 404 Mokhaberat, Kermanshah, Iran. Email: \\ s_komasi63@yahoo.com
}

Received 2021 July 23; Revised 2021 August 14; Accepted 2021 August 24.

\begin{abstract}
Background: The chronicity of multiple sclerosis (MS) plays a key role in $25 \%$ of suicides. Therefore, it is essential to assess the other influential factors in this regard.

objectives: The present study aimed to investigate the prevalence of suicidal thoughts and suicide attempts in patients with MS and the correlated factors.

Methods: This cross-sectional study was conducted on 203 MS patients referred to the MS Society of Kermanshah, Iran during August-October 2015. Data were collected using a checklist of demographics and patient records, Ifudu comorbidity index, 100meter walk test, and Beck's depression and suicidal thoughts scales. Data analysis was performed using binary logistic regression analysis.

Results: In total, $30 \%$ of the patients had suicidal thoughts, and $8.4 \%$ had suicide attempts. After adjustment for demographics, the obtained results indicated that illiterate patients $(\mathrm{P}<0.05)$ and those with a higher depression grade $(\mathrm{P}<0.001)$ were at a higher risk of suicidal thoughts. Illiteracy, older age at the first diagnosis, and being a housewife or unemployed $(\mathrm{P}<0.05)$ significantly decreased the likelihood of suicide attempts, while comorbidities, suicidal thoughts, and depression $(\mathrm{P}<0.05)$ significantly increased the likelihood of suicide attempts.

Conclusions: Since MS patients are highly prone to suicidal thoughts and even suicide attempts, providing training and preventive psychological interventions are recommended for high-risk cases, such as illiterate patients with challenging jobs, young patients, and patients with comorbidities and depression.
\end{abstract}

Keywords: Suicidal Ideation, Attempted Suicide, Depression, Comorbidity, Multiple Sclerosis

\section{Background}

Multiple sclerosis (MS) is a chronic autoimmune inflammatory disease, which affects approximately 728 thousand people in the United States (1) and 2.5 - 2.8 million people in the world $(2,3)$. According to a meta-analysis of the Iranian population in 2019, the prevalence of MS was estimated at 29.3 per 100,000 (4). The gradual progression of MS is associated with a series of physical and psychological complication, which affect the daily activities of the patients and reduce individual autonomy, psychological wellbeing, and quality of life (5). These issues may be followed by other adverse consequences, such as suicidal thoughts and even suicide attempts, and the risk of suicide increases within 2 - 20 years after diagnosis (6).

According to the literature, suicide attempts in MS pa- tients are 2 - 7.5 times more than the general population (7-10). The prevalence of suicidal thoughts and suicide attempts in these patients is reported to be $22.1-29.4 \%(11,12)$ and $3.7-6.4 \%(6,13)$, respectively. In addition, suicide accounts for $15 \%$ of deaths in MS patients (10). The chronicity of the disease plays a key role in $25 \%$ of suicides (14), and it seems that other factors are also influential in this regard.

Previous findings are contradictory regarding the predictive factors of suicidal thoughts and suicide attempts in MS patients. Meanwhile, most studies have suggested that variables such as young age $(2,12)$ or age of more than 65 years (11), low income, rapid disease progression, high levels of disability $(2,12)$, social isolation, depression $(2,12$, $13,15)$, hopelessness (15), and low self-efficacy (11) could predict suicidal thoughts. On the other hand, young age (16, 17) and male gender $(7,16,17)$ have been reported to predict 
the risk of suicide.

\section{Objectives}

Considering that MS patients experience suicidal thoughts with an increased risk of suicide attempts, the active assessment and screening of high-risk patients could be effective in designing preventive interventions (15). The present study aimed to evaluate the prevalence of suicidal thoughts and suicide attempts in patients with MS in the west of Iran, determined the demographic and clinical predictors of suicidal thoughts, and assess the demographic and clinical predictors of suicide attempts.

\section{Methods}

\subsection{Study Design and Inclusion Criteria}

This cross-sectional study was conducted on the patients diagnosed with MS at the MS Society of Kermanshah, Iran during August-October 2015. The patients were invited to participate in the study based on the inclusion criteria, which were as follows: (1) willingness to participate; (2) ability to communicate appropriately; (3) fluency in the Persian language; (4) age of 18 - 65 years; (5) not using psychiatric medication and (6) available medical records at the MS Society of Kermanshah. Patients would be excluded in case of fatigue due to commuting to the hospital and lack of coordination between their self-report data and medical records.

\subsection{Participants}

The sample size included 203 MS patients referred to the MS Society of Kermanshah within a three-month period. We invited all the recorded patients $(n=1,200)$, and only 261 patients were prepared to be enrolled in the study. Notably, 58 patients were excluded based on the eligibility criteria. In regression analysis, 15 cases must be available for each predictive variable, and our model contained 12 predictive variables. Therefore, the sample size of our study had to reach a minimum of 180 . Based on the formula of $\mathrm{N}>50+8 \mathrm{~m}$, the sample size had to be larger than $146(18)$.

\subsection{Instruments}

A checklist of patient data was used to collect selfreport data on age, gender, education level, occupation status, marital status, suicide attempts before and after MS diagnosis, and age at the time of suicide attempts at baseline. Other data were also recorded on disease history (clinical manifestations such as visual, motor, sensory, balance, and cognitive symptoms and their frequency), disease severity, functional impairment, and disability based on neurologist visits and medical records. Suicide attempt was defined as a self-directed, non-fatal behavior potentially undertaken to injure oneself with intent to die. In this study, the patients classified as those without a suicide attempt $(=1)$ and those with a suicide attempt $(=2)$.

Another research instrument used in our study was the 100-meter walk test, which is used to assess muscular dystrophy and shows the potential for expanded symptoms. The test is performed in a 20-meter square area without obstacles, and we provided the standard instructions to each patient, recommending travelling the 100 meters without stopping (19). In order to maintain the safety of the patients, a co-researcher walked alongside the patient until reaching the end of the route. If a patient reported a history of falling while walking, a gait belt would be fastened around their waist, and the guard would walk closely alongside the subject (20). Finally, the patients who were able to travel 100 meters without stopping were identified as code one, and the patients with disabilities were coded zero.

Ifudu comorbidity index was developed by Ifudu et al. in 1998 and is a degreed scale used to assess physical comorbidities. The scale has 14 components to evaluate the main body systems, including Q1 (ischemic heart disease), Q2 (other cardiovascular complications), Q3 (chronic respiratory diseases such as asthma), Q4 (autonomic neuropathy), Q5 (other neurological complications), Q6 (neuromuscular disorders), Q7 (infections such as HIV), Q8 (pancreatic and biliary diseases), Q9 (blood disorders), Q10 (back pain and spine/joint disorders), Q11 (visual impairments, reduced vision to total blindness), Q12 (organ disorders), Q13 (urogenital diseases), and Q14 (psychiatric diseases). Each item is scored from zero (no physical diseases) to three (severe disease) (21).

Beck depression inventory consists of 21 items, each of which is scored based on a three-point scale. The total score of the test is within the range of $0-63$. In this scale, the degree of depression is determined as follows: scores $0-4$ show the absence of depression, scores 5 - 9 indicate mild depression, scores 10 - 18 show mild-to-moderate depression, scores 19 - 29 indicate moderate-to-severe depression, and higher scores than 30 show severe depression. Beck et al. (22) estimated the test-retest reliability coefficient of the scale to be 0.93 .

Beck scale for suicide ideation is used to assess suicidal thoughts in the form of self-evaluation. The scale consists of 19 items to identify and measure the intensity of attitudes, behaviors, and planning to commit suicide. The main domains of this scale are death wish, active and inactive suicidal tendencies, duration and frequency of suicidal thoughts, level of self-control, preventive factors, and 
readiness to commit suicide. Beck and Steer (23) reported the validity of this scale to be $0.64-0.75$, and the Cronbach's alpha and test-retest reliability of the scale have been estimated at 0.97 and 0.54 , respectively. In the present study, scores 0 - 3 showed the absence of suicidal thoughts, and scores $\geq 4$ indicated suicidal thoughts in the MS patients.

\subsection{Data Collection}

After the approval of the study protocol by the Ethics Committee of Kermanshah University of Medical Sciences, eligible patients were invited to partake in the study by the administrator of the MS Society and referred to the research team with informed consent. After ensuring the patients about the anonymity and confidentiality of their information, demographic and medical data were collected using appropriate instruments.

Initially, the patients visited a neurologist, and after completing their demographics, medical history was also recorded, including data on clinical manifestations (visual, motor, sensory, balance, and cognitive symptoms) and their frequency, severity, functional impairment, and disability. At the next stage, the data were matched with the medical files of the patients at the MS Society to confirm the validity of the self-reported information. Following that, a general practitioner recorded the comorbidities of the patients using the Ifudu comorbidity index, and the 100-meter walk test was performed. Finally, the scales of depression and suicidal thoughts were provided to the patients by a clinical psychologist, and the necessary explanations were also given to complete the questionnaires. The patients were asked to answer all the questions. In case of illiteracy, the questions were read by the psychologist, and the responses were recorded.

\subsection{Statistical Analysis}

Data analysis was performed in SPSS version 20. Initially, binary logistic regression analysis was used to identify the correlations between the demographic and medical variables with suicidal thoughts. Following that, binary logistic regression analysis was repeated to determine the association of the mentioned variables (including suicidal thoughts) with suicide attempts. In addition, demographic characteristics such as age, gender, education level, and occupation status were adjusted in both stages. In all the statistical analyses, the P-value of less than 0.05 was considered significant. Before the statistical analysis, the statistical assumptions required for a regression analysis were evaluated, including normality, collinearity, and multicollinearity (18).

\section{Results}

The mean age of the participants was $36.2 \pm 10.2$ years, and $77.3 \%$ were female. In total, 203 patients were enrolled in the study, $30 \%$ of whom had suicidal thoughts, and $8.4 \%$ had attempted suicide. Table 1 shows the demographic and medical characteristics of the sample population. Accordingly, no significant differences were observed between the patients with and without suicidal ideation and suicide attempt in terms of age, gender, education level, and occupation status $(\mathrm{P}>0.05)$. Moreover, the patients with and without suicidal ideation and suicide attempt had no significant differences in terms of clinical factors such as the early symptoms of the disease $(\mathrm{P}=0.59,0.20)$, presentation frequency $(P=0.57,0.52)$, suicide attempt before MS diagnosis ( $P=0.21,0.67), 100$-meter walk test $(P=0.97,0.60)$, and comorbidities $(\mathrm{P}=0.08,0.09)$. Although no significant difference was denoted between the patients with and without suicidal ideation in terms of age upon the initial diagnosis $(\mathrm{P}=0.42)$, the patients with a suicide attempt were significantly younger upon the initial diagnosis $(\mathrm{P}<$ 0.02). Furthermore, the level of depression was higher in the patients with suicidal thoughts $(\mathrm{P}<0.001)$ and a suicide attempt $(P=0.012)$. Suicidal thoughts were also more prevalent in the patients who had attempted suicide $(\mathrm{P}<$ 0.001).

\subsection{Suicidal Thoughts}

Table 2 shows the adjusted odds ratio (OR), 95\% confidence interval, and P-value of each covariate included in the binary logistic regression model after adjustment for all the demographics (age, gender, education level, and occupation status). The indicators of the effect size showed proper explanatory power with respect to suicidal thoughts (Cox and Snell, $\mathrm{R}^{2}=0.391$; Nagelkerke, $\mathrm{R}^{2}=0.554$ ). Therefore, our model could explain $39.1-55.4 \%$ of the variance of suicidal thoughts.

Out of 11 covariates in the regression analysis, two were independently and significantly associated with suicidal thoughts. According to the information in Table 2, the patients with an education level below high school diploma were less likely to have suicidal thoughts compared to the illiterate patients $(\mathrm{OR}=0.06 ; \mathrm{P}=0.012)$ and those with a lower depression level $(\mathrm{OR}=1.16$; $\mathrm{P}<0.001)$. No significant differences were denoted between the patients with and without suicidal ideation in terms of age, gender, education level, occupation status, early symptoms of the disease, presentation frequency, suicide attempts before MS diagnosis, the 100-meter walk test, age upon early diagnosis, and comorbidities ( $\mathrm{P}>0.05)$. 


\begin{tabular}{|c|c|c|c|c|c|c|c|}
\hline Variables & $\begin{array}{c}\text { Total } \\
\text { Population, } \mathbf{n} \\
=\mathbf{2 0 3}\end{array}$ & $\begin{array}{c}\text { Non-suicidal } \\
\text { Thoughts, } n= \\
142(70.0 \%)\end{array}$ & $\begin{array}{c}\text { Suicidal } \\
\text { Thoughts, } \mathbf{n}= \\
\mathbf{6 1}(30.0 \%)\end{array}$ & P-Value $^{a, b}$ & $\begin{array}{c}\text { Non-suicidal } \\
\text { Attempts, } n= \\
186(91.6 \%)\end{array}$ & $\begin{array}{c}\text { Suicidal } \\
\text { Attempts, } n= \\
17(8.4 \%)\end{array}$ & P-Value $^{a, b}$ \\
\hline Age $($ mean \pm SD;y) & $36.25 \pm 10.22$ & $36.76 \pm 10.61$ & $35.06 \pm 9.22$ & 0.28 & $36.60 \pm 10.20$ & $32.41 \pm 9.92$ & 0.11 \\
\hline Gender $(\%)$ & & & & 0.76 & & & 0.49 \\
\hline Female & 77.3 & 76.7 & 78.7 & & 70.6 & 78.0 & \\
\hline Education level (\%) & & & & 0.41 & & & 0.68 \\
\hline Illiterate & 3.9 & 2.8 & 6.6 & & 3.8 & 5.9 & \\
\hline Below diploma & 36.0 & 38.0 & 31.1 & & 34.9 & 47.1 & \\
\hline High school diploma & 34.5 & 32.4 & 39.3 & & 35.5 & 23.5 & \\
\hline Academic & 25.6 & 26.8 & 23.0 & & 25.8 & 23.5 & \\
\hline Occupation status (\%) & & & & 0.58 & & & 0.41 \\
\hline Marketer & 11.8 & 11.3 & 13.1 & & 11.8 & 11.8 & \\
\hline Housewife & 54.2 & 53.5 & 55.7 & & 55.9 & 35.3 & \\
\hline Retired & 1.5 & 2.1 & 0.00 & & 1.6 & 0.0 & \\
\hline Unemployed & 26.1 & 25.4 & 27.9 & & 24.8 & 41.1 & \\
\hline First symptoms & & & & 0.59 & & & 0.20 \\
\hline None & 37.5 & 36.7 & 39.3 & & 37.7 & 35.3 & \\
\hline Visual symptoms & 25.1 & 22.5 & 31.1 & & 23.1 & 47.1 & \\
\hline Motor symptoms & 3.9 & 4.2 & 3.3 & & 4.3 & 0.0 & \\
\hline Sensory symptoms & 29.1 & 31.7 & 23.0 & & 30.1 & 17.6 & \\
\hline Balance symptoms & 4.4 & 4.9 & 3.3 & & 4.8 & 0.0 & \\
\hline Cognitive symptoms & 0.0 & 0.0 & 0.0 & & 0.0 & 0.0 & \\
\hline $\begin{array}{l}\text { Presentation frequency } \\
(\text { mean } \pm \text { SD })\end{array}$ & $1.87 \pm 1.65$ & $1.82 \pm 1.59$ & $1.97 \pm 1.80$ & 0.57 & $1.84 \pm 1.63$ & $2.12 \pm 1.90$ & 0.52 \\
\hline 100-meter walk test (\%) & 83.7 & 84.0 & 83.6 & 0.97 & 83.3 & 88.2 & 0.60 \\
\hline $\begin{array}{l}\text { Age upon initial diagnosis } \\
(\text { mean } \pm \text { SD;y) }\end{array}$ & $31.02 \pm 9.74$ & $31.39 \pm 9.96$ & $30.18 \pm 9.22$ & 0.42 & $31.51 \pm 9.53$ & $25.71 \pm 0.68$ & $0.018^{c}$ \\
\hline Comorbidities (mean \pm SD) & $3.46 \pm 3.01$ & $3.22 \pm 2.77$ & $4.03 \pm 3.46$ & 0.08 & $3.35 \pm 2.98$ & $4.65 \pm 3.14$ & 0.09 \\
\hline Depression (mean $\pm S D$ ) & $18.93 \pm 14.43$ & $13.32 \pm 10.94$ & $31.98 \pm 13.11$ & $0.001^{c}$ & $18.16 \pm 14.21$ & $27.35 \pm 14.52$ & $0.012^{c}$ \\
\hline $\begin{array}{l}\text { Suicidal thoughts (mean } \pm \\
\text { SD) }\end{array}$ & $4.17 \pm 6.96$ & $0.68 \pm 0.93$ & $12.29 \pm 8.09$ & $0.001^{c}$ & $3.52 \pm 6.07$ & $11.35 \pm 11.27$ & $0.001^{\mathrm{c}}$ \\
\hline
\end{tabular}

\subsection{Suicide Attempts}

The indicators of the effect size showed proper explanatory power with respect to suicide attempts (Cox and Snell, $\mathrm{R}^{2}=0.239$; Nagelkerke, $\mathrm{R}^{2}=0.546$ ). Therefore, our model could explain 23.9-54.6\% of the variance of suicide attempts. Table 2 also shows the 12 covariates in the regression analysis after adjustment for all the demographics, and six covariates were independently and significantly associated with suicide attempts. Furthermore, the information in Table 2 shows that illiteracy significantly increased the risk of suicide attempts compared to the education level of below high school diploma $(\mathrm{OR}=0.01 ; \mathrm{P}=0.007)$, high school education $(\mathrm{OR}=0.01 ; \mathrm{P}=0.002)$, and academic education $(\mathrm{OR}=0.01 ; \mathrm{P}=0.008)$.

According to the obtained results, younger age upon the initial diagnosis of $\mathrm{MS}(\mathrm{OR}=0.85 ; \mathrm{P}=0.018)$, increased comorbidities $(\mathrm{OR}=1.34 ; \mathrm{P}=0.022)$, higher depression levels $(\mathrm{OR}=1.12 ; \mathrm{P}=0.049)$, and more suicidal thoughts $(\mathrm{OR}=$ $1.20 ; \mathrm{P}=0.003)$ significantly increased the risk of committing suicide. However, housewives $(\mathrm{OR}=0.02 ; \mathrm{P}=0.019)$ and the unemployed patients $(\mathrm{OR}=0.02 ; \mathrm{P}=0.032)$ were at a lower risk of suicide attempts. No significant differences were observed between the patients with and without suicide attempts in terms of age, gender, early symptoms of the disease, presentation frequency, suicide attempts before MS diagnosis, and the 100-meter walk test $(\mathrm{P}>0.05)$. 


\begin{tabular}{|c|c|c|c|c|c|c|}
\hline Variables & Suicidal Thoughts (\%) & Adjusted OR & P-Value & Suicide Attempts (\%) & Adjusted OR & P-Value \\
\hline Age $(y)$ & - & $1.00(0.91-1.09)$ & 0.93 & - & $0.93(0.81-1.06)$ & 0.29 \\
\hline \multicolumn{7}{|l|}{ Gender (\%) } \\
\hline Female & 23.6 & Reference & & 7.6 & Reference & \\
\hline Male & 6.4 & $1.79(0.04-81.99)$ & 0.77 & 10.9 & $0.01(0.00-8.72)$ & 0.19 \\
\hline \multicolumn{7}{|l|}{ Education level (\%) } \\
\hline Illiterate & 2.0 & Reference & & 12.5 & Reference & \\
\hline Below diploma & 9.4 & $0.06(0.01-0.54)$ & $0.012^{\mathrm{b}}$ & 10.9 & $0.01(0.00-0.16)$ & $0.007^{\mathrm{b}}$ \\
\hline High school diploma & 11.7 & $0.16(0.02-1.37)$ & 0.10 & 5.7 & $0.01(0.00-0.06)$ & $0.002^{\mathrm{b}}$ \\
\hline Academic & 6.9 & $0.18(0.02-1.96)$ & 0.16 & 7.7 & $0.01(0.00-0.18)$ & $0.008^{b}$ \\
\hline \multicolumn{7}{|l|}{ Occupation status (\%) } \\
\hline Employee & 1.0 & Reference & & 15.4 & Reference & \\
\hline Marketer & 3.9 & $11.23(0.66-191.12)$ & 0.10 & 8.3 & $0.01(0.00-1.45)$ & 0.06 \\
\hline Housewife & 16.7 & $2.70(0.26-27.90)$ & 0.41 & 5.4 & $0.02(0.00-0.50)$ & $0.019^{b}$ \\
\hline Retired & 0.0 & $\mathrm{Na}$ & 0.99 & 0.0 & $\mathrm{Na}$ & 0.99 \\
\hline Unemployed & 8.4 & $2.28(0.20-26.19)$ & 0.51 & 13.2 & $0.02(0.00-0.72)$ & $0.032^{\mathrm{b}}$ \\
\hline \multicolumn{7}{|l|}{ Early symptoms } \\
\hline None & 11.7 & Reference & & 7.9 & Reference & \\
\hline Visual symptoms & 9.4 & $0.53(0.06-4.39)$ & 0.55 & 15.7 & $2.72(0.09-80.44)$ & 0.56 \\
\hline Motor symptoms & 1.0 & $3.82(0.26-55.55)$ & 0.33 & 0.0 & $\mathrm{Na}$ & 0.99 \\
\hline Sensory symptoms & 6.9 & $0.42(0.05-3.21)$ & 0.40 & 5.1 & $0.59(0.02-16.25)$ & 0.76 \\
\hline Balance symptoms & 1.0 & $0.86(0.07-11.05)$ & 0.91 & 0.0 & $\mathrm{Na}$ & 0.99 \\
\hline Cognitive symptoms & 0.0 & $\mathrm{Na}$ & 0.99 & 0.0 & $\mathrm{Na}$ & 0.99 \\
\hline Presentation frequency & - & $1.17(0.66-2.08)$ & 0.60 & - & $1.03(0.42-2.50)$ & 0.95 \\
\hline Suicide attempt before MS diagnosis (\%) & 2.0 & $1.99(0.24-16.12)$ & 0.52 & 12.5 & $1.30(0.09-19.64)$ & 0.85 \\
\hline 100-meter walk test (\%) & 25.1 & $1.39(0.38-5.12)$ & 0.62 & 8.8 & $1.81(0.18-17.93)$ & 0.61 \\
\hline Age upon initial diagnosis (y) & - & $0.97(0.89-1.05)$ & 0.39 & - & $0.85(0.75-0.97)$ & $0.018^{\mathrm{b}}$ \\
\hline Comorbidities & - & $1.00(0.86-1.17)$ & 0.95 & - & $1.34(1.04-1.72)$ & $0.022^{\mathrm{b}}$ \\
\hline Depression & - & $1.16(1.10-1.21)$ & $0.001^{*}$ & - & $1.12(1.03-1.98)$ & $0.049^{\mathrm{b}}$ \\
\hline Suicidal thoughts & - & - & - & - & $1.20(1.06-1.35)$ & $0.003^{\mathrm{b}}$ \\
\hline
\end{tabular}

${ }^{a}$ Variables listed in table were all considered as covariates in generating binary logistic regression model.

${ }^{\mathrm{b}}$ Significant odds ratio.

\section{Discussion}

The present study aimed to assess the prevalence of suicidal thoughts and suicide attempts in patients with MS and determine the predictive clinical and demographic factors in this regard. In line with previous studies $(6,11-$ 13 ), our findings indicated that $30 \%$ of the patients have suicidal thoughts, which resulted in suicide attempts in $8.4 \%$. Given that the prevalence of suicide is 10 per 100,000 in the general Iranian population (24), the results of the present study were indicative of the significantly higher prevalence rate of suicidal ideation and suicide attempts in MS patients.

The results of the present study showed no significant correlations between age, gender, early disease symptoms, presentation frequency, attempted suicide before MS diagnosis, and the 100-meter walk test with suicidal ideation and suicide attempts. In other words, none of these factors could explain suicidal thoughts and suicide attempts in patients with MS. Contrary to the results obtained by Pompili et al. (2), which indicated a correlation between disability and suicide in patients with MS, the lack of sig- 
nificance of the 100-meter walk test in our study showed that poorer physical performance due to muscular dystrophy has no effects on increased suicidal thoughts and suicide attempts. Furthermore, lack of significant differences between the patients with and without suicidal ideation and suicide attempts in terms of attempted suicide before disease diagnosis suggests that suicide attempts in MS patients may be due to the disease or factors such as depression. Our findings also demonstrated that the frequency of disease manifestations and presentations did not differ significantly between the patients with and without suicidal thoughts and suicide attempts. However, suicidal thoughts and suicide attempts may be influenced by the severity and speed of symptom progression and disability in MS patients. Further longitudinal studies are required to obtain more accurate results in this regard.

According to the results of the present study, the illiterate MS patients with higher levels of depression were at a significantly higher risk of suicidal thoughts and suicide attempts, which is consistent with previous studies $(2,12$, $13,15)$. Our findings regarding suicide attempts are also in line with the current literature $(2,12,16,17)$, indicating that older age upon the initial disease diagnosis and being a housewife/unemployed significantly decreased the risk of suicide attempts, while increasing comorbidities and suicidal thoughts, leading to a significantly higher risk of suicide attempts $(2,6,12,13,15,25)$.

Illiteracy is a contributing factor to suicidal thoughts and suicide attempts in MS patients since they tend to have a limited knowledge of the disease consequences and cannot independently read books, articles, and websites to find more information. On the other hand, health professionals in Iran provide patients with insufficient information about the consequences and management of the disease. This issue could lead to the confusion of patients, thereby making them feel they have limited control over the disease; consequently, lack of perceived control increases the risk of suicide (25).

Regarding the housewives and unemployed patients in the current research, it could be stated that this group of patients spend a great amount of time at home and are less involved with workplace challenges, are more related to other family members, and receive physical and psychological support; family support could effectively decrease suicidal tendencies (26).

As mentioned earlier, depression is highly common among MS patients and is a major trigger of suicidal thoughts and suicide attempts. Depression may also increase the disability of MS patients due to decreased functional ability (27). Depression directly and indirectly affects the outcome of individuals. According to Feinstein (13), approximately $29 \%$ of patients think about suicide, and severe depression could accurately predict $85 \%$ of suicide cases. Although the cause of the high rate of depressive symptoms in MS patients remains unknown, it is assumed that this issue stems from the biological changes in the brain, as well as the psychosocial stress stimulators and disabilities associated with chronic diseases (27). In MS, demyelination occurs in a part of the brain that controls feelings, thereby leading to the disruption of the treatment process, unpredictability of the disease attacks, reduced ability, and other complications (e.g., job loss). On the other hand, the adverse effects of the medications used in MS treatment could gradually cause depression in the patients (28). However, the risk factors for depression and suicide remain unclear in these patients.

According to the results of the present study, initial disease diagnosis at a younger age and more comorbidities also contributed to suicide attempts in the MS patients. Notably, young age upon the initial diagnosis was considered an independent risk factor $(2,7)$, which could be due to the unexpected nature of the disease. Young MS patients often experience short-term physical disability and dependence on wheelchair, as well as anxiety and depression in case of perceived threats (29), which is associated with the increased risk of suicide.

According to Gaskill et al. (25), the physical effects of MS increase the risk of suicidal thoughts and behavior. On the other hand, it seems that physical illnesses or other simultaneous psychiatric diseases may make the patients feel that they lack control over their disease (25). Since they experience more difficulties, they are commonly faced with misinterpretations regarding the nature of their disease (30). Such examples of these misconceptions are that MS invariably leads to failure, the inability to deal with loneliness and depression, and limited treatment options (29). These inefficient attitudes toward MS (especially in the presence of comorbidities) may intensify suicidal behavior in the patients.

\subsection{Strengths and Limitations}

Using standard instruments (e.g., 100-meter walk test) and assessment of comorbidities were the strengths of the present study. Meanwhile, our model could accurately explain $39.1-55.4 \%$ of the variance of suicidal thoughts and $23.9-54.6 \%$ of the variance of suicide attempts. The main limitations of our study were using predictive variables to increase the power of the model, small sample size, assessing the age of the patients at the time of suicide attempts based on the patients' reminders, the lengthy process of the interviews and questionnaire items, and investigating the patients in only one community (west of Iran). Finally, we did not examine the severity of the patients' disability by the expanded disability status scale and disease type 
(progressive/recurrent). Considering the possible effects of the mentioned limitations on the findings, it is recommended that further investigations assess these variables.

\subsection{Conclusions}

This study aimed to evaluate the prevalence of suicidal thoughts and suicide attempts in MS patients and determine the predictive factors of suicidal thoughts and suicide attempts. According to the results, $30 \%$ of the patients had suicidal thoughts, and $8.4 \%$ had attempted suicide. Illiteracy and depression were significant risk factors for suicidal thoughts and suicide attempts. On the other hand, older age upon the initial diagnosis and being a housewife/unemployed significantly decreased the risk of suicide attempts, while increased comorbidities and suicidal thoughts significantly increased the risk of suicide attempts. Since MS patients are highly prone to suicidal thoughts and even suicide attempts, providing effective training and preventive psychological interventions to high-risk patients, including illiterate patients with challenging jobs, young patients, and patients with comorbidities and depression, is recommended.

\section{Footnotes}

Authors' Contribution: Study concept and design: PS, PG, SK; Acquisition of data: PS; Analysis and interpretation of data: SK; Drafting of the manuscript: SK; Critical revision of the manuscript for important intellectual content: PS, PG; Statistical analysis: SK; Administrative, technical, and material support: PS, PG; Study supervision: PS.

Conflict of Interests: There was no conflict of interest.

Data Reproducibility: The data related to this study are available and if requested, present to the Journal Editor.

Ethical Approval: Ethical approval by Kermanshah University of Medical Sciences.

Funding/Support: The study was supported by Student Research Committee of Kermanshah University of Medical Sciences (grant No: 93481).

Informed Consent: We provided a written consent to the study participants and they consciously expressed their consent to participate in the study. This was done based on an official form of Kermanshah University of Medical Sciences and patients were assured that their identities and information would remain confidential. This study was based on Helsinki ethical standards.

\section{References}

1. Wallin MT, Culpepper WJ, Campbell JD, Nelson LM, Langer-Gould A, Marrie RA, et al. The prevalence of MS in the United States:
A population-based estimate using health claims data. Neurology. 2019;92(10):e1029-40. doi: 10.1212/WNL.0000000000007035. [PubMed: 30770430]. [PubMed Central: PMC6442006].

2. Pompili M, Forte A, Palermo M, Stefani H, Lamis DA, Serafini $\mathrm{G}$, et al. Suicide risk in multiple sclerosis: A systematic review of current literature. J Psychosom Res. 2012;73(6):411-7. doi: 10.1016/j.jpsychores.2012.09.011. [PubMed: 23148807].

3. Walton C, King R, Rechtman L, Kaye W, Leray E, Marrie RA, et al. Rising prevalence of multiple sclerosis worldwide: Insights from the Atlas of MS, third edition. Mult Scler. 2020;26(14):1816-21. doi: 10.1177/1352458520970841. [PubMed: 33174475]. [PubMed Central: PMC7720355].

4. Azami M, YektaKooshali MH, Shohani M, Khorshidi A, Mahmudi L. Epidemiology of multiple sclerosis in Iran: A systematic review and meta-analysis. PLoS One. 2019;14(4). e0214738. doi: 10.1371/journal.pone.0214738. [PubMed: 30964886]. [PubMed Central: PMC6456231].

5. Kolahkaj B, Zargar F. Effect of mindfulness-based stress reduction on anxiety, depression and stress in women with multiple sclerosis. Nurs Midwifery Stud. 2015;4(4). e29655. doi: 10.17795/nmsjournal29655. [PubMed: 26835467]. [PubMed Central: PMC4733502].

6. Stenager EN, Jensen B, Stenager M, Stenager K, Stenager E. Suicide attempts in multiple sclerosis. Mult Scler. 2011;17(10):1265-8. doi: 10.1177/1352458511401942. [PubMed: 21511689].

7. Bronnum-Hansen H, Stenager E, Nylev Stenager E, Koch-Henriksen N. Suicide among Danes with multiple sclerosis. J Neurol Neurosurg Psychiatry. 2005;76(10):1457-9. doi: 10.1136/jnnp.2004.056747. [PubMed: 16170097]. [PubMed Central: PMC1739366].

8. Coughlin SS, Sher L. Suicidal behavior and neurological illnesses.JDep Anx. 2013;9((Suppl 1)).

9. Fis K, Morehouse SA, Brown MG, Skedgel C, Murray TJ. Hospital-based psychiatric service utilization and morbidity in multiple sclerosis. Can J Neurol Sci. 1998;25(3):230-5. doi: 10.1017/s0317167100034065. [PubMed: 9706725].

10. Sadovnick AD, Eisen K, Ebers GC, Paty DW. Cause of death in patients attending multiple sclerosis clinics. Neurology. 1991;41(8):1193-6. doi: 10.1212/wnl.41.8.1193. [PubMed: 1866003].

11. Viner R, Patten SB, Berzins S, Bulloch AG, Fiest KM. Prevalence and risk factors for suicidal ideation in a multiple sclerosis population. J Psychosom Res. 2014;76(4):312-6. doi: 10.1016/j.jpsychores.2013.12.010. [PubMed: 24630182].

12. Turner AP, Williams RM, Bowen JD, Kivlahan DR, Haselkorn JK. Suicidal ideation in multiple sclerosis. Arch Phys Med Rehabil. 2006;87(8):10738. doi: 10.1016/j.apmr.2006.04.021. [PubMed: 16876552].

13. Feinstein A. An examination of suicidal intent in patients with multiple sclerosis. Neurology. 2002;59(5):674-8. doi: 10.1212/wnl.59.5.674. [PubMed: 12221156].

14. Fisher BJ, Haythornthwaite JA, Heinberg LJ, Clark M, Reed J. Suicidal intent in patients with chronic pain. Pain. 2001;89(2-3):199-206. doi: 10.1016/s0304-3959(00)00363-8. [PubMed: 11166476].

15. Lewis DS, Anderson KH, Feuchtinger J. Suicide prevention in neurology patients: Evidence to guide practice. $J$ Neurosci Nurs. 2014;46(4):241-8. doi: 10.1097/JNN.0000000000000062. [PubMed: 24992150]. [PubMed Central: PMC4083615].

16. Stenager EN, Stenager E, Koch-Henriksen N, Bronnum-Hansen H, Hyllested K, Jensen K, et al. Suicide and multiple sclerosis: An epidemiological investigation. J Neurol Neurosurg Psychiatry. 1992;55(7):5425. doi: 10.1136/jnnp.55.7.542. [PubMed: 1640228]. [PubMed Central: PMC489162].

17. Fredrikson S, Cheng Q, Jiang GX, Wasserman D. Elevated suicide risk among patients with multiple sclerosis in Sweden. Neuroepidemiology. 2003;22(2):146-52. doi: 10.1159/000068746. [PubMed: 12629281].

18. Pallant J. SPSS survival manual: A step by step guide to data analysis using SPSS for Windows (Version 12). 2nd ed. Australia: Allen \& Unwin; 2005. p. 142-52. 
19. Vadgama VK, Gharia R, Mehta K, Sanjiv R, Tripathi CB. A randomized, controlled clinical trial comparing efficacy, safety and cost effectiveness of lornoxicam with diclofenac sodium in patients of osteoarthritis knee. Internet J Medical Update. 2011;6(2). doi: 10.4314/ijmu.v6i2.68190.

20. Motl RW, Suh Y, Balantrapu S, Sandroff BM, Sosnoff JJ, Pula J, et al. Evidence for the different physiological significance of the 6and 2-minute walk tests in multiple sclerosis. BMC Neurol. 2012;12:6. doi: 10.1186/1471-2377-12-6. [PubMed: 22380843]. [PubMed Central: PMC3313866].

21. Ifudu O, Paul HR, Homel P, Friedman EA. Predictive value of functional status for mortality in patients on maintenance hemodialysis. Am J Nephrol.1998;18(2):109-16. doi:10.1159/000013318. [PubMed: 9569952].

22. Beck AT, Steer RA, Carbin MG. Psychometric properties of the Beck Depression Inventory: Twenty-five years of evaluation. Clin Psychol Rev. 1988;8(1):77-100. doi: 10.1016/0272-7358(88)90050-5.

23. Beck AT, Steer RA. Manual for the Beck Scale for suicide ideation. San Antonio, TX: Psychological Corporation; 1991.

24. Hassanian-Moghaddam $\mathrm{H}$, Zamani N. Suicide in Iran: The facts and the figures from nationwide reports. Iran J Psychiatry. 2017;12(1):73-7.
[PubMed: 28496505]. [PubMed Central: PMC5425355].

25. Gaskill A, Foley FW, Kolzet J, Picone MA. Suicidal thinking in multiple sclerosis. Disabil Rehabil. 2011;33(17-18):1528-36. doi: 10.3109/09638288.2010.533813. [PubMed: 21091136].

26. Long DD, Miller BJ. Suicidal tendency and multiple sclerosis. Health Soc Work. 1991;16(2):104-9. doi: 10.1093/hsw/16.2.104. [PubMed: 2071062].

27. Rintell DJ, Stazzone L, Guiod L, Gallagher H. Suicide, depression and MS: A newsletter for health professionals. 2. National Multiple Sclerosis Society; 2004.

28. Dehghan A, Mohammadkhan Kermanshahi S, Memarian R. Abundance of stress, anxiety and depression in multiple sclerosis patients. Alborz Univ Med J. 2013;2(2):82-8. doi:10.18869/acadpub.aums.2.2.82.

29. Janssens AJ, van Doorn PA, de Boer JB, van der Meché FG, Passchier J, Hintzen RQ. Perception of prognostic risk in patients with multiple sclerosis: The relationship with anxiety, depression, and diseaserelated distress. J Clin Epidemiol. 2004;57(2):180-6. doi: 10.1016/s08954356(03)00260-9.

30. Spring J, Beauregard N, Vorobeychik G. Multiple sclerosis: Myths and realities. British Columbia Med J. 2006;48(2):72. 